Muro de la Investigación, 2021(1), enero-junio

ISSN: 2523-2886

DOI: https://doi.org/10.17162/rmi.v6i1.1434

\title{
Estrategias de afrontamiento y bienestar psicológico en estudiantes del nivel secundario
}

\section{Coping strategies and psychological well-being in secondary school students}

\author{
Ninet Gutierrez Flores ${ }^{1 \mathrm{a}}$ Yanní Sherly Veliz Llaja² y \\ Dámaris Susana Quinteros Zúñiga ${ }^{3}$ \\ Universidad Peruana Unión, Perú ${ }^{123}$
}

Recibido: 15 de mayo de 2020

Aceptado: 19 de diciembre de 2020

\section{Resumen}

El objetivo del presente estudio fue analizar la relación entre las estrategias de afrontamiento y bienestar psicológico en estudiantes del nivel secundario de una institución particular de Lima Sur. Se empleó el muestreo no probabilístico y la muestra estuvo conformada por 216 adolescentes, de ambos sexos cuyas edades oscilaron entre 12 y 17 años. El diseño es no experimental, de corte transversal y alcance correlacional. Se utilizó la Escala de Afrontamiento para adolescentes (ACS) elaborado por Frydenberg y Lewis (1993) y la Escala de Bienestar Psicológico de Ryff, elaborado por Ryff (1989). Los resultados obtenidos muestran que existe correlación entre el bienestar psicológico y las estrategias de afrontamiento: concentrarse en resolver el problema (chi=43.448, $\mathrm{p}=.000)$, esforzarse y tener éxito (chi $=46.797^{\mathrm{a}}, \mathrm{p}=.000$ ), fijarse en lo positivo ( $\mathrm{chi}=47.263^{\mathrm{a}}, \mathrm{p}=.000$ ), buscar apoyo espiritual (chi $\left.=22.360^{\mathrm{a}}, \mathrm{p}=.004\right)$, buscar ayuda profesional $\left(\mathrm{chi}=22.360^{\mathrm{a}}, \mathrm{p}=.004\right)$, preocuparse (chi $\left.=26.931^{\mathrm{a}}, \mathrm{p}=.000\right)$, falta de afrontamiento $\left(\mathrm{chi}=30.789^{\mathrm{a}}, \mathrm{p}=.000\right)$, reducción de la tensión (chi $\left.=43.297^{\mathrm{a}}, \mathrm{p}=.000\right)$, reservarlo para sí $\left(\mathrm{chi}=26.614^{\mathrm{a}}, \mathrm{p}=.001\right)$ y autoinculparse $\left(\mathrm{chi}=39.270^{\mathrm{a}}\right.$, $\mathrm{p}=.000)$.

Palabras Claves: Estrategias de afrontamiento, bienestar psicológico, adolescencia, estrés.

\begin{abstract}
The objective of the present study was to determine the relationship between coping strategies and psychological well-being in students at the secondary level of a particular institution in South Lima. Non-probabilistic sampling was used and the sample consisted of 216 adolescents, of both sexes whose ages range from 12 to 17 years. The design is non-experimental, crosssectional and correlational in scope. The adolescent coping scale (ACS) prepared by Erica Frydenberg and Ramón Lewis (1993) and Ryff's psychological well-being scale, developed by Carol Riff (1989), were used. The results obtained show that there is a correlation between the coping strategies: concentrate on solving the problem $\left(\mathrm{chi}=43.448^{\mathrm{a}}, \mathrm{p}=.000\right)$, strive and succeed (chi $\left.=46.797^{\mathrm{a}}, \mathrm{p}=.000\right)$, look at the positive (chi $\left.=46.797^{\mathrm{a}}, \mathrm{p}=.000\right)$, seek spiritual support $\left(\mathrm{chi}=22.360^{\mathrm{a}}, \mathrm{p}=.004\right)$, seek professional help $\left(\mathrm{chi}=22.360^{\mathrm{a}}, \mathrm{p}=.004\right)$, worry $\left(\mathrm{chi}=26.931^{\mathrm{a}}\right.$,
\end{abstract}

${ }^{\mathrm{a} C o r r e s p o n d e n c i a ~ a l ~ a u t o r: ~}$

E-mail: ninetflores@upeu.edu.pe 
$\mathrm{p}=.000)$, lack of coping $\left(\mathrm{chi}=30.789^{\mathrm{a}}, \mathrm{p}=.000\right)$, reduction of tension $\left(\mathrm{chi}=43.297^{\mathrm{a}}, \mathrm{p}=.000\right)$, reserve for yourself $\left(\mathrm{chi}=26.614^{\mathrm{a}}, \mathrm{p}=.001\right)$ and self-incrimination $\left(\mathrm{chi}=39.270^{\mathrm{a}}, \mathrm{p}=.000\right)$.

Keywords: coping strategies, psychological well-being, adolescence, stress.

\section{Introducción}

Según la Organización Mundial de la Salud (OMS, 2014a), aproximadamente 16 millones de adolescentes del sexo femenino-entre 15 a 19 años-y 1 millón de niñasmenores de 15 años — salen embarazadas cada año, la mayoría de ellas pertenecen a países en vías de desarrollo. Por otro lado, la misma organización reportó (2016) que 2’000,000 homicidios son cometidos por jóvenes cuyas edades oscilan entre 10 a 29 años, constituyendo un $43 \%$ del total de homicidios en el mundo.

Por su parte, el Instituto Nacional de Estadística y Geografía (INEGI, 2013) reseñó que la edad promedio en la que los jóvenes en México reportan haber tomado su primera bebida alcohólica es a los 16 años, y durante el 2011 fallecieron un total de 38 mil jóvenes en accidentes de tránsito bajo los efectos del alcohol. Desde otra perspectiva, Perú no es ajeno a una realidad critica para los adolescentes, ya que se han reportado 31,893 casos de VIH, siendo el 4.8\% de ellos, menores de 19 años (UNICEF, 2014). El Instituto Nacional de Estadística e Informática (INEI, 2014) reportó que el embarazo adolescente se incrementó al 14.6\%. por otr lado, el diario La República (2014) también indicó que, en los últimos cinco años, los actos delictivos cometidos entre los jóvenes de 12 y 23 años incrementaron al menos en un $80 \%$.

En este contexto, la OMS (2016) señala—según sus últimas investigaciones—que la depresión es la principal causa de mortalidad y el suicidio es la tercera causa en las personas. Estas dificultades y la carencia de adecuadas estrategias de afrontamiento, hacen que se desarrollen situaciones de riesgo, desencadenando una problemática en sus vidas. Entonces, se hace preciso enfatizar la presencia de factores decisivos en el uso de estrategias de afrontamiento de problemas de salud mental. Respecto a esto, Castaño y León del Barco (2010) 
sostienen que, aquellas personas que se consideran a sí mismas como extravertidas, cálidas y sociables, son las que tienden a utilizar con más frecuencia estrategias activas, buscando el contacto con otras personas y así compartir sus experiencias (positivas o negativas) y expresar sus emociones.

Por otro lado, Figueroa, et al. (2005) encontraron que los adolescentes con un nivel bajo de bienestar psicológico, no utilizan adecuadas estrategias de afrontamiento. Sin embargo, aquellos jóvenes que evidenciaron un bienestar psicológico alto, utilizan estrategias adecuadas de afrontamiento, como: concentrarse en resolver el problema, esforzarse y tener éxito, preocuparse, invertir en amigos íntimos y distracción física. Asimismo, Antonovsky et al. (1979) afirman que el control correcto de la tensión frente a los problemas, determinan un resultado exitoso y una adecuada condición de bienestar. Por su parte, Mikkelsen (2009) señala la importancia de identificar los problemas de los jóvenes y cómo los afrontan, favoreciendo un mejor ajuste en su estado de bienestar y satisfacción en sus vidas.

En este sentido, y basándose en la importancia del asunto en los adolescentes, el objetivo de esta investigación fue comprobar la relación demostrativa entre diversas estrategias de afrontamiento y el respectivo bienestar emocional en adolescentes estudiantes del nivel secundario de una institución educativa particular de Lima Sur, 2017.

\section{Metodo}

Para la selección de los participantes se utilizó el método no probabilístico por conveniencia. La muestra estuvo compuesta por 216 estudiantes, con edades comprendidas entre los 12 y 17 años, de ambos sexos, que están realizando sus estudios en el Colegio Adventista Miraflores. En la tabla 1, se muestra que el 51.9\% son varones, asimismo el 73.1\% está entre 12 y 14 años y que la mayoría de los estudiantes profesan la religión adventista $(49.1 \%)$ 
Tabla 1

Análisis de frecuencia de las características de los participantes

\begin{tabular}{clcc}
\hline Variables & & $\mathrm{N}$ & $\%$ \\
\hline \multirow{3}{*}{ Sexo } & Femenino & 104 & 48.1 \\
& Masculino & 112 & 51.9 \\
\multirow{3}{*}{ Edad } & $12-14$ & 158 & 73.1 \\
& $15-17$ & 58 & 26.9 \\
& & & \\
\multirow{3}{*}{ Grado } & Primer año & 53 & 24.5 \\
& Segundo año & 48 & 22.2 \\
& Tercer año & 51 & 23.6 \\
& Cuarto año & 32 & 14.8 \\
Religión & Quinto año & 32 & 14.8 \\
& & & \\
& Católico & 39 & 18.1 \\
& Adventista & 106 & 49.1 \\
& Evangélico & 13 & 6 \\
& Otros & 58 & 26.9 \\
\hline
\end{tabular}

En cuanto a la evaluación de la variable de Estrategias de Afrontamiento se utilizó la Escala denominada Adolescent Coping Scales (ACS. Frydenberg y Lewis, 1993), como base de adaptación en escolares de Lima Metropolitana. El instrumento se aplica de manera grupal o individual con una duración aproximada de 15 minutos. Puede ser administrado en adolescentes entre los 12 y 18 años de edad. El instrumento está compuesto por 80 ítems, con respuestas tipo Likert que van desde nunca lo hago (1) hasta lo hago con mucha frecuencia (5). Asimismo, la prueba está dividida en tres dimensiones y cada dimensión tiene aproximadamente de 5 a 7 estrategias, las dimensiones son: resolver el problema, referencia a otros y afrontamiento no productivo. Según la validación hecha por Canessa (2002), la confiabilidad del instrumento es altamente significativa, llegando a obtener una consistencia interna que osciló entre .54 y .84 en su forma frecuente, y las confiabilidades test/re-test, que señalan las subescalas que varían de .44 a .84 .

Por otro lado, para la evaluación del bienestar psicológico (Ryff, 1989), se tomó la prueba adaptada por Van Dierendonck, et al. (2006), denominada Escala de Bienestar Psicológico de Ryff, la cual paso por criterio de jueces obteniendo una adecuada validez 0.66 
y una confiabilidad de 0.88 . El cuestionario se aplica de manera individual o grupal a adolescentes, durante 15 minutos aproximadamente. Esta prueba se divide en seis dimensiones claramente diferenciadas: relaciones positivas, autoaceptación, dominio del entorno, crecimiento personal, autonomía y propósito en la vida. Cada una de ellas se compone de un número de ítems entre 4 y 6.

\section{Resultados}

\section{Descriptivos}

\section{Niveles de uso de las estrategias de afrontamiento}

En la tabla 2 se observa, el $29.6 \%$ de estudiantes del nivel secundario emplean con mucha frecuencia la estrategia de diversiones relajantes, asimismo el $51.4 \%$ de estos adolescentes utilizan a menudo la estrategia esforzarse y tener éxito, además el $56.5 \%$ en ocasiones usan la estrategia de hacerse ilusiones, también el $48.6 \%$ de los participantes eligen raras veces la falta de afrontamiento; y por último $32.9 \%$ del alumnado no utiliza la estrategia reducción de la tensión.

Tabla 2

Niveles de uso de los estilos de afrontamiento en adolescentes

\begin{tabular}{|c|c|c|c|c|c|c|c|c|c|c|}
\hline \multirow[t]{2}{*}{ Estrategia } & \multicolumn{2}{|c|}{ No usada } & \multicolumn{2}{|c|}{ Raras veces } & \multicolumn{2}{|c|}{ Algunas veces } & \multicolumn{2}{|c|}{ A menudo } & \multicolumn{2}{|c|}{$\begin{array}{l}\text { Con mucha } \\
\text { frecuencia }\end{array}$} \\
\hline & $\mathrm{n}$ & $\%$ & $\mathrm{n}$ & $\%$ & $\mathrm{n}$ & $\%$ & $\mathrm{n}$ & $\%$ & $\mathrm{n}$ & $\%$ \\
\hline $\begin{array}{l}\text { Resolver el } \\
\text { problema }\end{array}$ & 1 & $0.5 \%$ & 11 & $5.1 \%$ & 85 & $39.4 \%$ & 100 & $43.3 \%$ & 19 & $8.8 \%$ \\
\hline Esforzarse & 0 & 0 & 10 & $4.6 \%$ & 58 & $26.9 \%$ & 111 & $51.4 \%$ & 37 & $17.1 \%$ \\
\hline Invertir en amigos & 6 & $2.8 \%$ & 39 & $18.1 \%$ & 90 & $41.7 \%$ & 73 & $33.8 \%$ & 8 & $3.7 \%$ \\
\hline Pertenencia & 0 & 0 & 12 & $5.6 \%$ & 107 & $49.5 \%$ & 91 & $42.1 \%$ & 6 & $2.8 \%$ \\
\hline Fijarse positivo & 2 & $0.9 \%$ & 16 & $7.4 \%$ & 90 & $41.7 \%$ & 89 & $41.2 \%$ & 19 & $8.8 \%$ \\
\hline $\begin{array}{l}\text { Diversiones } \\
\text { relajantes }\end{array}$ & 1 & $0.5 \%$ & 14 & $6.5 \%$ & 32 & $14.8 \%$ & 105 & $48.6 \%$ & 64 & $29.6 \%$ \\
\hline Distracción física & 14 & $6.5 \%$ & 31 & $14.4 \%$ & 37 & $17.1 \%$ & 79 & $36.6 \%$ & 55 & $25.5 \%$ \\
\hline Apoyo social & 5 & $2.3 \%$ & 44 & $20.4 \%$ & 98 & $45.4 \%$ & 58 & $26.9 \%$ & 11 & $5.1 \%$ \\
\hline Acción social & 2 & $0.9 \%$ & 49 & $22.7 \%$ & 85 & $39.4 \%$ & 56 & $25.9 \%$ & 24 & $11.1 \%$ \\
\hline Apoyo espiritual & 4 & $1.9 \%$ & 26 & $12 \%$ & 69 & $31.9 \%$ & 83 & $38.4 \%$ & 34 & $15.7 \%$ \\
\hline Ayuda profesional & 14 & $6.5 \%$ & 40 & $23.1 \%$ & 84 & $38.9 \%$ & 49 & $22.7 \%$ & 19 & $8.8 \%$ \\
\hline Preocuparse & 0 & 0 & 8 & $3.7 \%$ & 72 & $33.3 \%$ & 103 & $47.7 \%$ & 33 & $15.3 \%$ \\
\hline
\end{tabular}




\begin{tabular}{lcccccccccc} 
Hacerse ilusiones & 3 & $1.4 \%$ & 32 & $14.8 \%$ & 122 & $56.5 \%$ & 55 & $25.5 \%$ & 4 & $1.9 \%$ \\
$\begin{array}{l}\text { Falta de } \\
\text { afrontamiento }\end{array}$ & 28 & $13 \%$ & 105 & $48.6 \%$ & 71 & $32.9 \%$ & 12 & $5.6 \%$ & 0 & 0 \\
$\begin{array}{l}\text { Ignorar el } \\
\text { problema }\end{array}$ & 26 & $12 \%$ & 77 & $35.6 \%$ & 89 & $41.2 \%$ & 23 & $10.6 \%$ & 1 & $0.5 \%$ \\
$\begin{array}{l}\text { Reducción de la } \\
\text { tensión }\end{array}$ & 71 & $32.9 \%$ & 92 & $42.6 \%$ & 43 & $19.9 \%$ & 10 & $4.6 \%$ & 0 & $0 \%$ \\
$\begin{array}{l}\text { Reservárselo } \\
\text { Autoinculparse }\end{array}$ & 7 & $3.2 \%$ & 56 & $25.9 \%$ & 103 & $47.7 \%$ & 44 & $20.4 \%$ & 6 & $2.8 \%$ \\
\hline
\end{tabular}

\section{Nivel de bienestar psicológico}

En la tabla 3 se observa que el $25.5 \%$ de participantes presentan un nivel alto de bienestar psicológico, mientras que el $49.5 \%$ se ubica en un nivel medio. Por otro lado, respecto a las dimensiones de bienestar psicológico, el 32.9\% evidencia un nivel alto de autonomía, el 49.1\% alcanzó un nivel medio respecto al propósito en la vida y el 39.4\% mostró un nivel bajo en las relaciones positivas.

\section{Tabla 3}

Nivel de bienestar psicológico

\begin{tabular}{lcccccc}
\hline & \multicolumn{2}{c}{ Bajo } & \multicolumn{2}{c}{ Medio } & \multicolumn{2}{c}{ Alto } \\
\cline { 2 - 7 } & $\mathrm{n}$ & $\%$ & $\mathrm{~N}$ & $\%$ & $\mathrm{n}$ & $\%$ \\
\hline $\begin{array}{l}\text { Bienestar } \\
\text { psicológico }\end{array}$ & 54 & $25 \%$ & 107 & $49.5 \%$ & 55 & $25.5 \%$ \\
$\begin{array}{l}\text { Autoaceptación } \\
\text { Relaciones }\end{array}$ & 83 & $38.4 \%$ & 65 & $30.1 \%$ & 68 & $31.5 \%$ \\
$\begin{array}{l}\text { positivas } \\
\text { Autonomía }\end{array}$ & 85 & $39.4 \%$ & 65 & $30.1 \%$ & 66 & $30.6 \%$ \\
$\begin{array}{l}\text { Dominio del } \\
\text { entorno }\end{array}$ & 62 & $33.3 \%$ & 73 & $33.8 \%$ & 71 & $32.9 \%$ \\
$\begin{array}{l}\text { Crecimiento } \\
\text { personal }\end{array}$ & 59 & $27.3 \%$ & 94 & $43.5 \%$ & 63 & $29.2 \%$ \\
$\begin{array}{l}\text { Propósito en la } \\
\text { vida }\end{array}$ & 60 & $27.8 \%$ & 89 & $41.2 \%$ & 67 & $31 \%$ \\
\hline
\end{tabular}

\section{Resultados inferenciales}

Correlación entre las estrategias de afrontamiento y bienestar psicológico 
En la tabla 4, se observa que de acuerdo a la prueba estadística no paramétrica Chicuadrada de Pearson, indica que el valor de p (sig.) es menor a .005 lo cual indica que existe asociación entre las variable bienestar psicológico y las siguientes estrategias de afrontamiento: concentrarse en resolver el problema $\left(\mathrm{chi}=43.448^{\mathrm{a}}, \mathrm{p}=.000\right)$, esforzarse y tener éxito (chi $=46.797^{\mathrm{a}}, \mathrm{p}=.000$ ), fijarse en lo positivo $\left(\mathrm{chi}=47.263^{\mathrm{a}}, \mathrm{p}=.000\right.$ ), buscar apoyo espiritual $\left(\mathrm{chi}=22.360^{\mathrm{a}}, \mathrm{p}=.004\right)$, buscar ayuda profesional $\left(\mathrm{chi}=22.360^{\mathrm{a}}, \mathrm{p}=.004\right)$, preocuparse (chi=26.931 a,$p=.000)$, falta de afrontamiento $\left(\mathrm{chi}=30.789^{\mathrm{a}}, \mathrm{p}=.000\right)$, reducción de la tensión $\left(\mathrm{chi}=43.297^{\mathrm{a}}, \mathrm{p}=.000\right)$, reservarlo para sí $\left(\mathrm{chi}=26.614^{\mathrm{a}}, \mathrm{p}=.001\right)$ y autoinculparse $\left(\mathrm{chi}=39.270^{\mathrm{a}}\right.$, $\mathrm{p}=.000)$.

\section{Tabla 4}

Tabla de correlación entre las estrategias de afrontamiento y bienestar psicológico

\begin{tabular}{lccc}
\hline Estrategias & Chi-cuadrado & Gl & $\mathrm{p}$ \\
\hline Concentrarse en resolver el problema & $43.448^{\mathrm{a}}$ & 8 &, 000 \\
Esforzarse y tener éxito & $46.797^{\mathrm{a}}$ & 6 &, 000 \\
Fijarse en lo positivo & $47.263^{\mathrm{a}}$ & 8 &, 000 \\
Buscar diversiones relajantes & $47.263^{\mathrm{a}}$ & 8 &, 010 \\
Distracción física & $15.714^{\mathrm{a}}$ & 8 &, 047 \\
Invertir en amigos íntimos & $13.532^{\mathrm{a}}$ & 8 &, 095 \\
Buscar pertenencia & $12.700^{\mathrm{a}}$ & 6 &, 048 \\
Buscar apoyo social & $18.928^{\mathrm{a}}$ & 8 &, 015 \\
Acción social & $8.125^{\mathrm{a}}$ & 8 &, 421 \\
Buscar apoyo espiritual & $22.360^{\mathrm{a}}$ & 8 &, 004 \\
Buscar ayuda profesional & $22.360^{\mathrm{a}}$ & 8 &, 004 \\
Preocuparse & $26.931^{\mathrm{a}}$ & 6 &, 000 \\
Hacerse ilusiones & $15.478^{\mathrm{a}}$ & 8 &, 050 \\
Falta de afrontamiento & $30.789^{\mathrm{a}}$ & 6 &, 000 \\
Ignorar el problema & $16.752^{\mathrm{a}}$ & 8 &, 033 \\
Reducción de la tensión & $43.297^{\mathrm{a}}$ & 6 &, 000 \\
Reservarlo para si & $26.614^{\mathrm{a}}$ & 8 &, 001 \\
Autoinculparse & $39.270^{\mathrm{a}}$ & 8 &, 000 \\
\hline
\end{tabular}

Cierto número de especialistas mencionan que, ante las estrategias improductivas, no existen estrategias de afrontamiento mejores ni peores. Antes bien, debería analizarse su 
eficacia en función de algunos factores importantes: la situación generadora de estrés; el momento vital de la persona; los objetivos que persiguen con determinado comportamiento; el apoyo social, entre otros (Romero, et al., 2010). Esto guarda estrecha relación a lo indicado por Contini, et al. (2003) quienes mencionan que el temor que pueda sentir este grupo de estudio ante su futuro, hace que la estrategia "preocuparse", se relacione a un adecuado bienestar psicológico.

Esto se entiende como el móvil de acciones específicas en el futuro; por lo que es probable que las estrategias de evitación no sean disfuncionales, sino que dependan de la naturaleza del agente estresor frente al cual se despliega esta estrategia (Rodríguez et al., 1993). Se indica, además, que la estrategia "reducción de la tensión", evidencia el intento de sentirse mejor por medio de actividades que disminuyan el estrés que ocasiona el problema por el cual se atraviesa (Figueroa, et al., 2005). Se observa que la poca utilización de esta estrategia se relaciona con un nivel elevado de bienestar psicológico. Acciones como la ingesta de alcohol, sustancias psicoactivas, comportamientos de huida temporaria de la casa o de la escuela; no suelen formar parte de las acciones que toman los adolescentes para alcanzar un adecuado bienestar psicológico.

\section{Referencias}

Antonovsky, A. (1979). Health, stress and coping. San Francisco: Jossey-Bass. Recuperado de: http://trove.nla.gov.au/work/7249690?selectedversion=NBD1463125

Berger, K. (2006). Psicología del desarrollo, Infancia y adolescencia. (7 $7^{\mathrm{a}}$ ed). Madrid: Editorial Medica Panamericana.

Castaño, E \& León del Barco, B. (2010). Estrategias de afrontamiento del estrés y estilos de conducta interpersonal. International Journal of Psychology and Psychological Therapy, 10(2), 245-257. Recuperado de: http://www.ijpsy.com/volumen10/num2/260/estrategias-de-afrontamiento-del-estrsES.pdf

Casullo, M. y Castro, A. (2000). Evaluación del bienestar psicológico en estudiantes adolescentes argentinos. Revista de Psicología de la PUCP, 18(2), 35-68. Recuperado de: http://revistas.pucp.edu.pe/index.php/psicologia/article/view/6840

Contini, N., Coronel, P., Levin, M. \& Estevez, A. (2003). Estrategias de afrontamiento y bienestar psicológico en adolescentes escolarizados de Tucumán. Revista de Psicología 
de la PUCP, 11(1), 179-200. Recuperado de: http://revistas.pucp.edu.pe/index.php/psicologia/article/view/3723/3705

Fernández, E. (1997). Estilos y estrategias de afrontamiento. Cuaderno de prácticas de motivación y emoción. Madrid: Pirámide.

Figueroa, I., Contini, N., Lacunza, B. \& Levin, M. (2005). Las estrategias de afrontamiento y su relación con el nivel de bienestar psicológico: un estudio con adolescentes de nivel socioeconómico bajo de Tucumán (Argentina). Anales de Psicología, 21(1), 66-72. Recuperado de: https://search.proquest.com/openview/dd4a90e809c87fed332aa466578c091b/1?pqorigsite $=$ gscholar $\& \mathrm{cbl}=1606360$

Frydenberg, E. \& Lewis, R. (1991). Adolescent coping: The different ways in which boys and girls cope. Journal of Adolescence, 14, 119-133. Recuperado de: WwW.sciencedirect.com/science/article/pii/014019719190025M

Frydenberg, E. \& Lewis, R. (1996). ACS. Escalas de Afrontamiento para Adolescentes. Manual. Madrid: TEA.

González, R., Montoya, I., Casullo, M. \& Bernabéu, J. (2002). Relación entre estilos y estrategias de afrontamiento y bienestar psicológico en adolescentes. Psicothema. 14 (2), 363-369. Recuperado de: http://www.psicothema.com/psicothema.asp?id=733

INEGI: Instituto Nacional de Estadística y Geografía. (2013). Estadísticas a propósito del día internacional de la juventud. Recuperado de: http://www.cinu.mx/minisitio/juventud_2013/Juventud_INEGI.pdf

Mikkerlsen, F. (2009). Satisfacción con la vida y estrategias de afrontamiento en un grupo de adolescentes universitarios en Lima (Tesis de Licenciatura). Pontificia Universidad Católica del Perú Recuperado de: http://tesis.pucp.edu.pe/repositorio/handle/123456789/416

Organización Mundial de la Salud. (2014a). El embarazo en la adolescencia. Recuperado de: http://www.who.int/mediacentre/factsheets/fs364/es/

Ryff, C. (1989). Happiness is verything, or is it? Exploration sin the meanin of psycological. Journal of social Psychology, 57(6) 1069-1081. Recuperado de: https://www.google.com.pe/url?sa=t\&rct=j\&q=\&esrc=s\&source=web\&cd=1\&cad=rja \&uact=8\&sqi=2\&ved=0ahUKEwj7 x6qOgIzTAhVBfpAKHYtEA3IQFggYMAA\&url $=$ http $\% 3 \mathrm{~A} \% 2 \mathrm{~F} \% 2$ Faging.wisc.edu\%2Fpdfs\%2F379.pdf\&usg=AFQjCNEbw3vwrxPj UHVjng5TrolHaGb66w\&sig2=jKCxEHZdAZDztGlDKbkbow\&bvm=bv.151426398, d.Y2I 